\title{
PD-L1 Expression on Tumor Cells Is Associated With a Poor Outcome in a Cohort of Caucasian Nasopharyngeal Carcinoma Patients
}

\author{
Christoph Minichsdorfer ${ }^{1 \dagger}$, Felicitas Oberndorfer ${ }^{2 \dagger}$, Christoph Krall ${ }^{3}$, Gabriela Kornek ${ }^{1}$, \\ Leonhard Müllauer ${ }^{2}$, Christina Wagner ${ }^{1}$ and Thorsten Fuereder ${ }^{1 *}$ \\ ${ }^{1}$ Department of Internal Medicine I \& Comprehensive Cancer Center, Medical University of Vienna, Vienna, Austria, \\ ${ }^{2}$ Department of Pathology, Medical University of Vienna, Vienna, Austria, ${ }^{3}$ Center for Medical Statistics, Informatics and \\ Intelligent Systems, Medical University of Vienna, Vienna, Austria
}

\section{OPEN ACCESS}

Edited by:

Dietmar Thurnher,

Medical University of Graz, Austria

Reviewed by:

Sjoukje F. Oosting, University Medical Center

Groningen, Netherlands Jens Bentzen

Herlev Hospital, Denmark

${ }^{*}$ Correspondence:

Thorsten Fuereder

thorsten.fuereder@meduniwien.ac.at

tThese authors have contributed equally to this work

Specialty section

This article was submitted to

Head and Neck Cancer,

a section of the journal

Frontiers in Oncology

Received: 08 August 2019 Accepted: 14 November 2019 Published: 29 November 2019

Citation:

Minichsdorfer $C$, Oberndorfer $F$

Krall C, Kornek G, Müllauer L,

Wagner $C$ and Fuereder T (2019)

PD-L1 Expression on Tumor Cells is Associated With a Poor Outcome in a Cohort of Caucasian Nasopharyngeal Carcinoma Patients. Front. Oncol. 9:1334. doi: 10.3389/fonc.2019.01334
Background: Nasopharyngeal carcinoma (NPC) is endemic in East Asia but rare in the western world. Programmed death ligand 1 (PD-L1) expression on NPC correlates with clinical outcomes. However, data for Caucasian NPC patients are missing. Thus, we performed this retrospective analysis for investigating the potential association of immune checkpoint protein expression with outcome parameters in Caucasian NPC patients.

Methods: Fifty-five patients with NPC treated between 1993 and 2018 at the Medical University of Vienna were identified. After the exclusion of Asian patients, data on baseline demographic, tumor stage, overall survival (OS), and disease-free survival (DFS) of 30 patients were analyzed. Their tumor samples were stained and scored (low vs. high) for PD-L1, programmed death receptor 1 (PD-1), lymphocyte activating gene 3 (LAG3), and cluster of differentiation 8 (CD8) antibodies. Statistical analysis was performed with Kaplan-Meier plots and log-rank test. Estimated hazard ratios of dichotomized analysis were calculated, together with 95\% confidence intervals and $p$-values of Wald tests.

Results: PD-L1 expression was $\geq 50 \%$ in 6 (20\%) patients, whereas 19 (63\%) had $\geq 1 \%$ expression and 5 (17\%) tumor samples were PD-L1-negative. While sex and age had no impact on DFS or OS, $<50 \%$ PD-L1 expression on tumor cells (TC) was associated with a significantly longer OS (log rank test $p=0.037 ; \mathrm{HR} 0.275 ; 95 \% \mathrm{Cl}$ 0.073-1.03). There was no influence on DFS (log rank test $p=0.34 ; \mathrm{HR} 0.599 ; 95 \%$ $\mathrm{Cl}$ 0.208-1.728). However, <10\% PD-L1 expression on tumor infiltrating lymphocytes (TILs) was correlated with a worse DFS (log rank test $p=0.0057 ; \mathrm{HR} 4.06 ; 95 \% \mathrm{Cl}$ 1.389-11.868). LAG3 expression or the number of TILs did not play any prognostic role in our population.

Conclusion: The PD-L1 expression rate on Caucasians was comparable to that in Asian patients. Although these results have to be interpreted with caution due to the limited number of Caucasian patients available, our data suggest that $\geq 50 \%$ PD-L1 expression on TC is associated with a poor outcome, while $\geq 10 \%$ PD-L1 expression on TILs is correlated with improved DFS. A prospective biomarker analysis of a predefined Caucasian NPC subpopulation would be desirable in future trials.

Keywords: nasopharyngeal carcinoma, PD-L1, PD-1, LAG-3, Caucasian population 


\section{INTRODUCTION}

The incidence of nasopharyngeal carcinoma (NPC) varies drastically between the Caucasian and Asian populations in Europe/the USA and certain parts of Asia. The incidence in the western world lies between 0.5 and 2 cases per 100,000 people; therefore, NPC is regarded as an orphan disease in these countries. By contrast, NPC is endemic to southern China and Hong Kong with an incidence reaching 25 cases per 100,000 (1-3). In the endemic regions NPC is mainly associated with Epstein Barr virus (EBV) infection, whereas in the USA and Europe, alcohol and tobacco use are the major risk factors for the development of NPC $(4,5)$. The World Health Organization distinguishes three histological subtypes of this disease. Type I is the keratinizing squamous cell carcinoma, which represents the sporadic form. The non-keratinizing subtypes are further divided into types II and III (differentiated and undifferentiated non-keratinizing carcinoma, respectively). Type III is strongly associated with EBV infection and accounts for almost 95\% of NPC in the endemic regions of south-east Asia (6-8). Irrespective of the subtype, treatment for intermediate and locally advanced NPC comprises concurrent chemoradiation preceded or followed by systemic chemotherapy, according to the NCCN guidelines (9, 10). Of note, survival outcomes and response to chemoradiation markedly differ between Asian and non-Asian patients, most likely reflecting the differences underlying tumor biology between the two groups, as demonstrated in a recent populationbased analysis (11).

In an attempt to improve the prognosis of locally advanced NPC, intensive research efforts focusing on the evolving landscape of NPC immuno-oncology have been made during the last couple of years. NPC is regarded as a highly immunogenic tumor characterized by high rates of tumor infiltrating lymphocytes (TILs) $(12,13)$. Furthermore, preclinical investigations suggest that EBV-driven NPC cells up-regulate critical immune checkpoint proteins including programmed death ligand 1 (PD-L1) (14). Clinical data on the potential significance of PD-L1 overexpression in NPC are conflicting; while some studies have proposed a detrimental effect of elevated PD-L1 expression on outcome parameters, others have shown a positive association of PD-L1 expression with survival in NPC patients (15-18). These studies, however, were conducted solely in Asian populations. Neither clinical data nor analyses characterizing the infiltration of TILs and the role of PD-L1, PD-1, or alternative checkpoints proteins such as LAG3 in Caucasian NPC patients that take into account the distinct tumor biology of this population are available so far. The clinical impact of the PD-1/PDL1 axis and LAG3 on the outcome of Caucasian NPC is currently unclear.

Based on this background, we performed the present retrospective analysis in order to investigate the expression levels of PD-L1 on TC and PD-1 and LAG3 on TILs in a nonAsian NPC patient population and subsequently evaluate the correlation of checkpoint protein expression with overall survival (OS) and disease-free survival (DFS).

\section{PATIENTS AND METHODS}

\section{Data Collection}

Patients eligible for this single center retrospective analysis had histologically confirmed NPC diagnosed between 1st January 1993 and 30th September 2018 at the Medical University of Vienna. A total of 55 patients were identified. Out of this, histological specimens were available from 37 patients. Two patients were lost to follow up and therefore unavailable for our analysis. An additional five patients were excluded according to the study plan due to their Asian background. Therefore, 30 cases were found to be eligible. All 30 patients received a combination of radiotherapy (R) (66-72 Gray) and chemotherapy (CHT), either as concurrent radiochemotherapy (RCHT), neoadjuvant $\mathrm{CHT}$, or adjuvant CHT. Survival analysis was monitored in September 2018.

The study was performed in accordance with the Declaration of Helsinki and good clinical practice guidelines and was approved by the local ethics committee (EK 1416/2017).

\section{Tissue Analysis}

Samples of histologically proven, formalin fixed, paraffin embedded tissue of nasopharyngeal squamous cell carcinoma were examined retrospectively. For histopathological reports, EBV status was assessed by in-situ hybridization (using Ventana EBER Probe 800-2842, Roche Diagnostics GmbH, Mannheim, Germany or Bond EBER Probe PB0589, Leica Biosystems, Nussloch, Germany) using an automated BenchMark Ultra, Roche/Ventana or Leica BOND III, Leica Biosystems immunostainer, respectively. The patients were either biopsied or they underwent surgical treatment at the Medical University of Vienna, Department of Otorhinolaryngology, Head and Neck Surgery. Archived hematoxylin/eosin stained slides were re-evaluated by an experienced pathologist, and the most representative area for each case was chosen. Representative areas were marked and measured and were of dimensions of at least 6 and $25 \mathrm{~mm}^{-2}$ for biopsies and for surgical resections, respectively.

\section{Immunohistochemistry (IHC)}

IHC analysis was performed on freshly cut $3 \mu \mathrm{m}$ thick serial sections of the selected formalin fixed, paraffin embedded tissues. IHC staining was conducted on automated immunostainers (BenchMark Ultra, Roche/Ventana and Leica BOND III, Leica Biosystems) with suitable positive and negative controls. The commercially available antibodies PD-L1 (clone 22C3, mouse monoclonal, Dako, CA, USA, dilution 1:50) and LAG3 (clone 17B4, mouse monoclonal, LifeSpan BioSciences Inc., WA, USA, dilution 1:100) were employed for estimating expression levels of PD-L1 and LAG3, respectively. IHC assays were carried out according to the manufacturer's instructions, after antigen recovery with heat-induced epitope retrieval (HIER) in Cell Conditioning 1 (CC1) buffer (Ventana Medical Systems, AZ, USA) using a standardized in-house protocol. IHC staining of PD1 (clone 315M-96, mouse monoclonal, Cell Marque, CA, USA, dilution 1:50) and CD8 (clone M7103, mouse monoclonal, Dako, 
CA, USA, dilution 1:100) were conducted on a Leica BOND III automated stainer using a standardized in-house routine protocol with HIER for 20 min with BOND Epitope Retrieval Solution 1 (Leica Biosystems, Nussloch, Germany).

The slides were examined independently by two experienced pathologists and a consensus diagnosis was ascertained. Each IHC staining was analyzed semi-quantitatively in a selected representative area.

TABLE 1 | Patient characteristics $(n=30)(100 \%)$.

\begin{tabular}{|c|c|}
\hline Sex & $n(\%)$ \\
\hline Female & $6(20)$ \\
\hline Male & $24(80)$ \\
\hline \multicolumn{2}{|l|}{ Age (years) } \\
\hline Median & 56 \\
\hline Range & $22-88$ \\
\hline T-stage & $n(\%)$ \\
\hline $\mathrm{T} 1$ & $5(17)$ \\
\hline T2 & $10(33)$ \\
\hline T3 & $4(13)$ \\
\hline T4 & $11(37)$ \\
\hline \multicolumn{2}{|l|}{$\mathrm{N}$-stage } \\
\hline No & $5(17)$ \\
\hline $\mathrm{N} 1$ & $2(7)$ \\
\hline N2 & $19(63)$ \\
\hline N3 & $4(13)$ \\
\hline \multicolumn{2}{|c|}{ American Joint Committee on Cancer (AJJC) 7 stage } \\
\hline 1 & $1(4)$ \\
\hline$\|$ & $3(10)$ \\
\hline III & $13(43)$ \\
\hline IVa & $13(43)$ \\
\hline \multicolumn{2}{|l|}{ EBV status } \\
\hline Positive & $19(63)$ \\
\hline Negative & $11(37)$ \\
\hline WHO type & $n(\%)$ \\
\hline I & $2(7)$ \\
\hline$\|$ & $12(40)$ \\
\hline III & $16(53)$ \\
\hline \multicolumn{2}{|l|}{ DFS (months) } \\
\hline Median & 36.5 \\
\hline Range & $1-174$ \\
\hline \multicolumn{2}{|l|}{ OS (months) } \\
\hline Median & 54 \\
\hline Range & $1-184$ \\
\hline Treatment & $n(\%)$ \\
\hline Induction chemotherapy & $7(23)$ \\
\hline Primary radiotherapy & $6(20)$ \\
\hline Radiochemotherapy & $20(67)$ \\
\hline Adjuvant chemotherapy & $6(20)$ \\
\hline Surgery/radiation & $3(10)$ \\
\hline Recurrence of disease & $13(43)$ \\
\hline
\end{tabular}

Please note that treatment modalities add up to more than $100 \%$, since some patients received multiple types of therapy.
Based on the total number of TC in the selected area, the percentage of TC showing specific PD-L1 expression was estimated. Since no validated cut-off values for PD-L1 positivity have been published so far for NPC, we adopted the published cut-offs used in the KEYNOTE-040 study for head and neck squamous cell carcinoma (HNSCC). This was possible, especially since we employed the same antibody clone (i.e., 22C3 from Dako), which was used in the KEYNOTE-040 trial (19). According to these suggested cut-offs, PD-L1-positive membranous staining on a TC proportion of $<1 \%$ was graded as negative, $\geq 1-<50 \%$ was graded as low, and $\geq 50 \%$ as high at any intensity (19).

IHC expression of PD-L1, PD-1, LAG3, and CD8 on TILs was evaluated as the proportion (percentage) of the tumor infiltrating inflammatory cells.

No validated standards have been determined for PD-L1 positivity on TILs in NPC patients. Therefore, the classification system of the phase II POPLAR trial in lung cancer and the protocol described by Karpathiou et al. in HNSCC were adopted $(20,21)$ and PD-L1-positive TILs were divided into four groups and three grades $(<1 \%=$ group $0=$ negative, $1-5 \%=$ group $1=$ low, $5-10 \%=$ group $2=$ low, and $\geq 10 \%=$ group $3=$ high).

No validated cut-off values for LAG-3, PD-1, and CD8 on TILs in NPC samples are established. Therefore, LAG-3, CD8, and PD-1 expression rates for every single patient were determined and the median expression score calculated. Subsequently, all NPC samples were divided into three subgroups according to the aforementioned median of LAG-3 and CD8 immunostaining (adapted as described previously) (22). This scoring method was also employed for PD-1, both for consistency and due to the lack of established PD-1 cut-off values. LAG-3 was graded

TABLE 2 | Immunohistochemical characterization of NPCs studied here.

\begin{tabular}{ll}
\hline PD-L1 expression on tumors & $\boldsymbol{n}$ (\%) \\
\hline High ( $\geq 50 \%)$ & $6(20)$ \\
Low $(\geq 1$ to $<50 \%)$ & $19(63)$ \\
Negative (<1\%) & $5(17)$ \\
PD-L1 expression on TILs & \\
High ( $\geq 10 \%)$ & $23(77)$ \\
Low ( $\geq 1$ to $<10 \%)$ & $6(20)$ \\
Negative (<1\%) & $1(3)$ \\
Lag3 expression & \\
High (>5\%) & $15(50)$ \\
Low ( $\geq 1$ to $\leq 5 \%)$ & $13(43)$ \\
Negative (<1\%) & $2(7)$ \\
PD-1-positive TILs & \\
High (>1\%) & $12(40)$ \\
Low (0 to 1\%) & $9(30)$ \\
Negative (0\%) & $9(30)$ \\
CD8-positive TILs & \\
High (<35\%) & $10(33)$ \\
Low (1 to 35\%) & $20(67)$ \\
Negative (0\%) & $0(0)$
\end{tabular}


negative when $0 \%$, low when $1-5 \%$, and high when $>5 \%$ immunoreactive TILs were detected. Similarly, CD8 was graded negative when $0 \%$, low when $1-35 \%$, and high when $>35 \%$ immunoreactive lymphocytes were detected. PD-1 expression on TILs was grouped into negative $(0 \%)$, low $(0-1 \%)$, and $\operatorname{high}(>1 \%)$.

For survival analysis, the expression of PD-L1 on TC was categorized into a high $(\geq 50 \%)$ and a low/negative group $(<50 \%)$. Additionally, TILs were grouped according to their PD-L1 expression into low/negative $(<10 \%)$ and high $(\geq 10 \%)$ groups. Similarly, we categorized TILs into low/negative $(\leq 1$ and $\leq 5 \%)$ and high ( $>1$ and $>5 \%)$ groups, based on their PD-1 and LAG3 expression.

\section{Statistical Analysis}

To investigate the effects of potential factors for survival, KaplanMeier plots for each level were generated and a log rank test was performed. For factors with levels "negative," "low" and "high", the analysis was repeated after pooling the classes "negative" and "low" together. Estimated hazard ratios of this dichotomized analysis have been tabled together with $95 \%$ confidence intervals and $p$-values of Wald tests. Due to the small number of patients in the study, no control for multiple testing was performed. A two-sided $p$-value $<0.05$ was considered statistically significant.

\section{RESULTS}

\section{Patient Characteristics}

The demographic and clinical baseline characteristics of the patients involved in this study are depicted in Table 1. The median age of the patients was 56 years (range 22-88 years). Six $(20 \%)$ patients were female and $24(80 \%)$ male. While only 19 (63\%) patients had EBV-positive disease, the predominant histological subtypes were type II $(n=12[40 \%])$ and type III $(n=16[53 \%])$. The distribution of the disease stage, according to the 7th American Joint Committee on Cancer (AJCC) edition, was as follows: I with $n=1(4 \%)$, II with $n=3(10 \%)$, III with $n$ $=13(43 \%)$, and IVa with $n=13(43 \%)$.

Induction chemotherapy (ICHT) was recommended for 7 patients $(23 \%)$, of which $6(20 \%)$ received primary radiation and $1(3 \%)$ experienced a lethal ICHT-related adverse event. Twenty (67\%) patients were treated with primary RCHT. Adjuvant chemotherapy following RCHT was administered only in a minority of $6(20 \%)$ patients. Three (10\%) patients were treated with multimodality surgery/radiation approaches. Recurrent disease after primary curative therapy was recorded in 13 (43\%) patients.

The median DFS and OS were 36.5 months (range 1-174 months) and 54 months (1-184 months), respectively.

A minority of 6 (20\%) patients had tumors with high PDL1 expression, 19 (63\%) had a low expression, and $5(17 \%)$

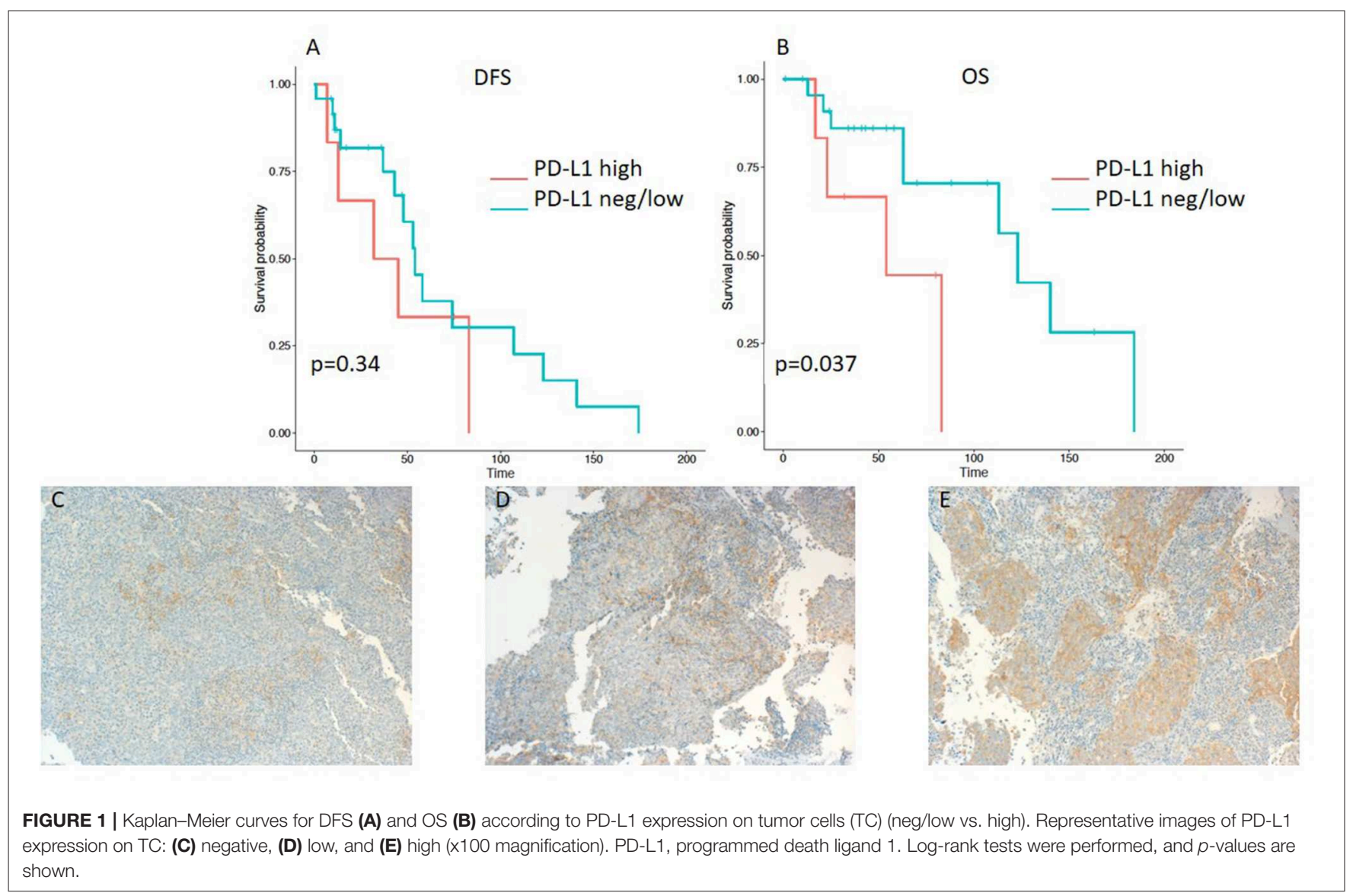



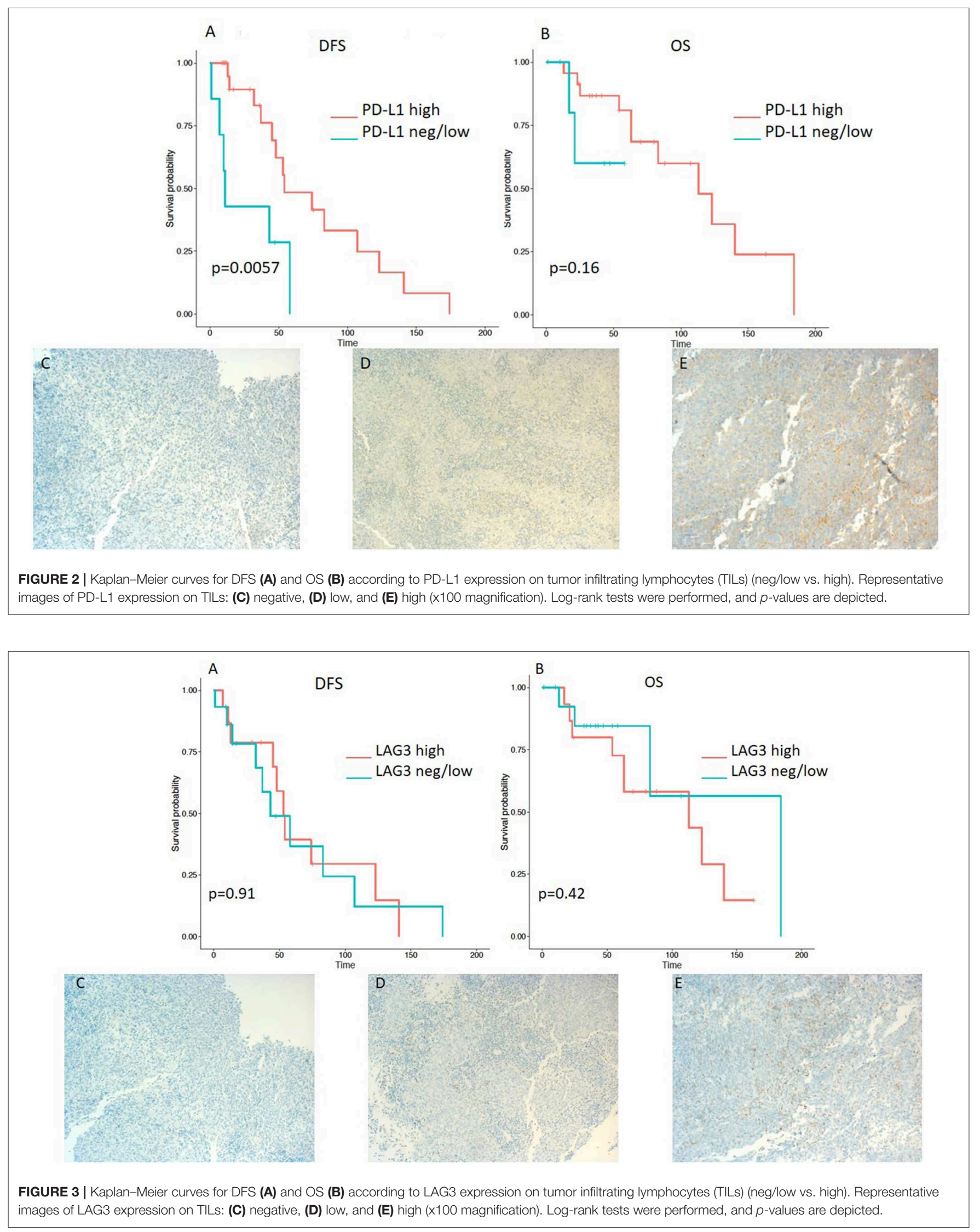

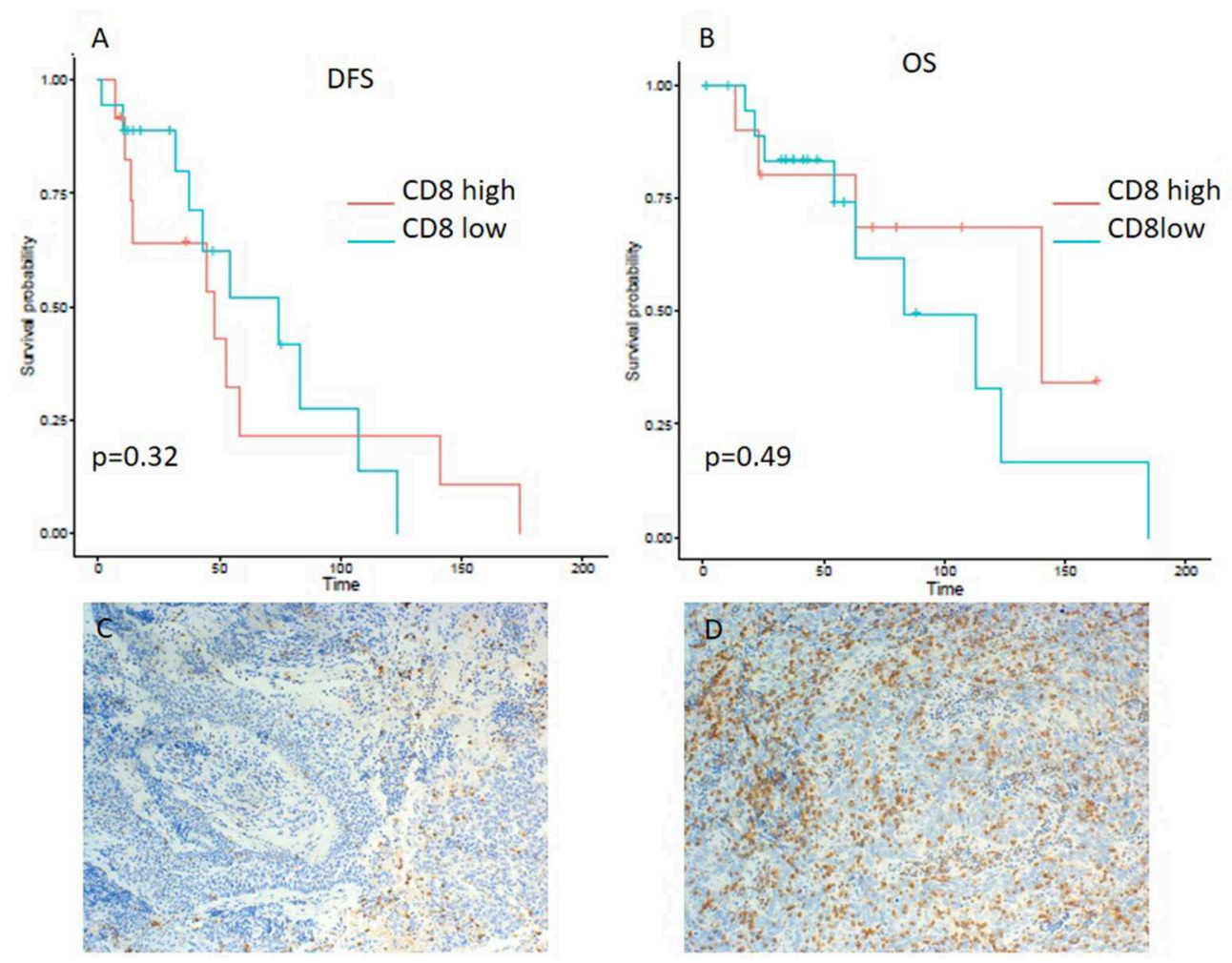

FIGURE 4 | Kaplan-Meier curves for DFS (A) and OS (B) according to CD8 expression on tumor infiltrating lymphocytes (TILs) (low vs. high). Representative images of CD8 expression on TILs: (C) low and (D) high (x100 magnification). Log-rank tests were performed, and p-values are depicted.

tumor samples were PD-L1-negative (Table 2). PD-L1 on TILs was highly positive in $23(77 \%)$ patients, low in $6(20 \%)$ cases, and negative in 1 (3\%) sample. PD-1 expression on TILs was high in $12(40 \%)$ NPC patients, low in $9(30 \%)$ samples, and negative in $9(30 \%)$ cases. CD8-positive TILs were high in $10(33 \%)$ and low in $20(67 \%)$ cases. TILs expressing high levels, low levels, and no LAG3 were detected in 15 (50\%), 13 (43\%), and 2 (7\%) patients, respectively (Table 2 ). Representative examples of PDL1, PD-1, LAG3, and CD8 expressing TC or TILs are depicted in Figures 1C-E, 2C-E, 3C-E, 4C,D, 5C-E.

\section{Association Between Checkpoint Inhibitor Protein Expression Level and Survival}

Patients with $\leq 50 \%$ PD-L1 expression on tumor samples had a significantly longer OS in the Kaplan-Meier log-rank test analysis ( $p=0.037)$, although the hazard ratio for OS did not reach a significant level (HR 0.275; 95\% CI 0.073-1.03; $p=0.055$ ) (Table 3). Interestingly, there was no difference in DFS (HR $0.599 ; 95 \%$ CI $0.208-1.728 ; p=0.343$ and log-rank test: $p=0.34$ ) (Table 3 and Figures 1A,B).

On the other hand, lower expression of PD-L1 on TILs $(<10 \%)$ was associated with a shorter DFS, as evident from a logrank test $(p=0.0057)$. Additionally, a significant difference in hazard ratios between these two classes was identified employing a Wald test in a Cox proportional-hazards model (HR 4.06; 95\% CI 1.389-11.868; $p=0.01$ ) (Table 3). In contrast to the OS impact of PD-L1 expression on TC, no difference in OS was detected for PD-L1 expression on TILs (Figures 2A,B). Likewise, PD-1 expression on TILs had no significant influence on OS (HR 0.356; 95\% CI $0.106-1.197 ; p=0.095$ ) or on DFS (HR 0.889; 95\% CI 0.347-2.277; $p=0.806$ ), as depicted in Table 3. Apart from that, no difference in DFS or OS for negative/low and high LAG3 expressing patients was detected (HR 1.055; 95\% CI 0.426-2.615; $p=0.907$; log-rank test: $p=0.91$; and HR 0.577; 95\% CI 0.1522.191; $p=0.419$; log-rank test: $p=0.42$. respectively) (Table 2 and Figures 3A,B). We further analyzed potential associations of CD8 expression and PD-1 on TILs with survival. However, none of these factors had a significant influence on OS (HR 1.538; 95\% CI $0.451-5.248$; $p=0.49$ for CD8 and HR 0.356; 95\% CI $0.106-$ $1.197 ; p=0.095$ for PD-1) or DFS (HR 1.672; 95\% CI $0.598-$ $4.674 ; p=0.327$ for CD8 and HR 0.889; 95\% CI 0.347-2.277; $p$ $=0.806$ for PD-1) (Table 3 and Figures 4A,B, 5A,B).

\section{DISCUSSION}

In this retrospective study, we highlight a potential association between tumor PD-L1 expression on TC and decreased OS in a Caucasian cohort of NPC patients. Our study shows that, surprisingly, a higher rate of PD-L1-positive TILs is correlated with a longer DFS.

The presently available literature investigating the expression of PD-L1 on both TC and TILs in NPC 

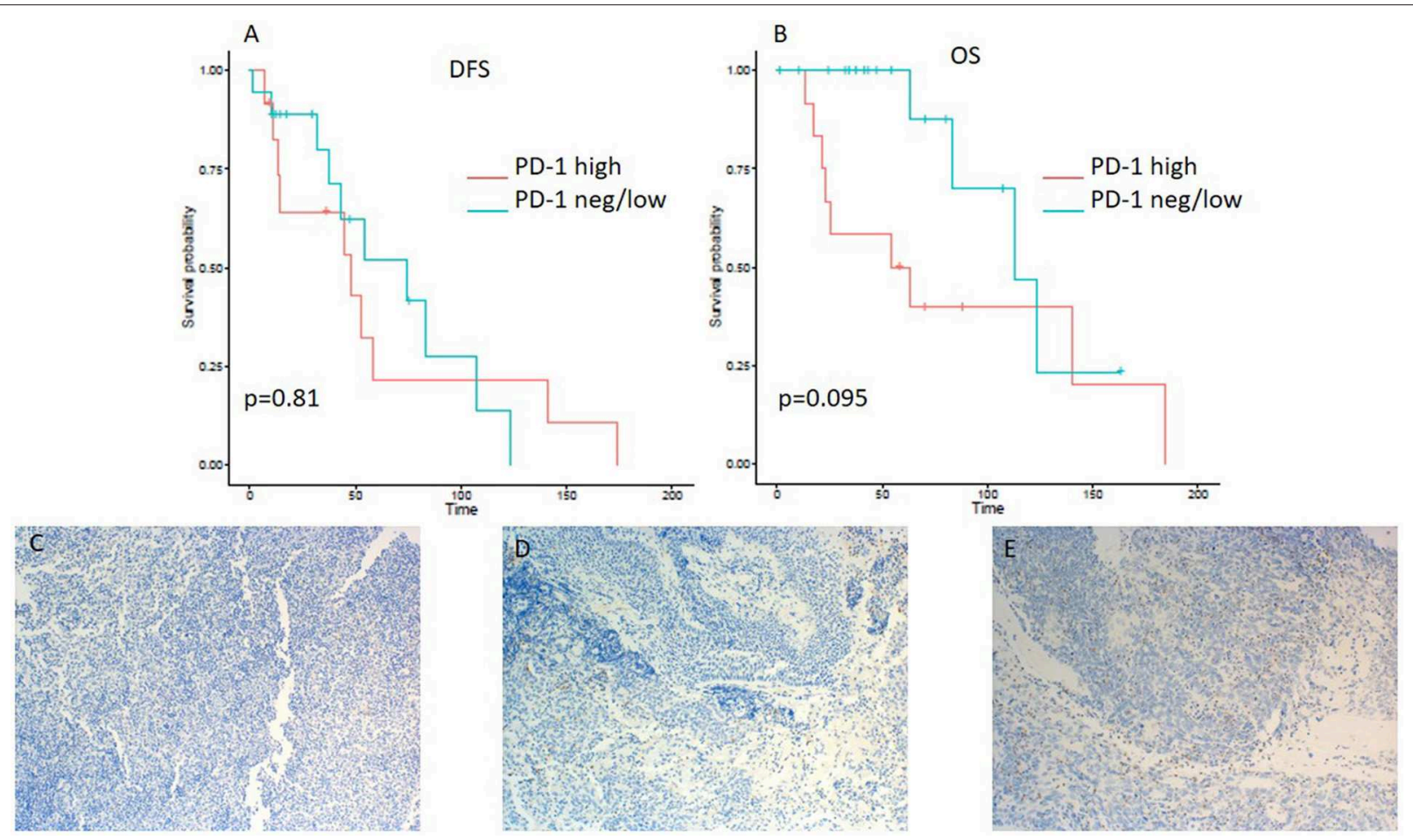

FIGURE 5 | Kaplan-Meier curves for DFS (A) and OS (B) according to PD-1 expression on tumor infiltrating lymphocytes (TILs) (neg/low vs. high). Representative images of PD-1 expression on TILs: (C) negative, (D) low, and (E) high (x100 magnification). Log-rank tests were performed, and $p$-values are depicted.

TABLE 3 | Association of DFS and OS with clinical and pathological characteristics.

\begin{tabular}{lll}
\hline & HR (95\% Cl) & p-value \\
\hline DFS & & \\
Gender & $2.601(0.882-7.672)$ & 0.083 \\
T-stage (T1/2 vs. T3/4 & $1.536(0.614-3.841)$ & 0.359 \\
N-stage (N0/1 vs. N2/3) & $0.436(0.15-1.266)$ & 0.127 \\
EBV (pos vs. neg) & $0.994(0.388-2.548)$ & 0.99 \\
PD-L1 tumor (neg/low vs. high) & $0.599(0.208-1.728)$ & 0.343 \\
PD-L1 IC (neg/low vs. high) & $4.06(1.389-11.868)$ & $\mathbf{0 . 0 1}$ \\
LAG3 (neg/low vs. high) & $1.055(0.426-2.615)$ & 0.907 \\
CD8 grading (high vs. low) & $1.672(0.598-4.674)$ & 0.327 \\
PD-1 (neg/low vs. high) & $0.889(0.347-2.277)$ & 0.806 \\
OS & & \\
Gender & $1.668(0.43-6.476)$ & 0.46 \\
T-stage (T1/2 vs. T3/4 & $2.292(0.676-7.772)$ & 0.183 \\
N-stage (N0/1 vs. N2/3) & $0.319(0.079-1.285)$ & 0.108 \\
EBV (pos vs. neg) & $1.99(0.524-7.553)$ & 0.312 \\
PD-L1 tumor (neg/low vs. high) & $0.275(0.073-1.03)$ & 0.055 \\
PD-L1 IC (neg/low vs. high) & $3.192(0.57-17.895)$ & 0.187 \\
LAG3 (neg/low vs. high) & $0.577(0.152-2.191)$ & 0.419 \\
CD8 grading (high vs. low) & $1.538(0.451-5.248)$ & 0.492 \\
PD-1 (neg/low vs. high) & $0.356(0.106-1.197)$ & 0.095 \\
\hline Fstmateg & & \\
\hline & & \\
\hline & & \\
\hline
\end{tabular}

Estimated hazard ratios of this dichotomized analysis are tabled together with 95\% confidence intervals and $p$-values of Wald tests. Bold value indicates a significant difference.

patients focuses solely on patients in endemic areas and, therefore, these reports uniformly come from Asian cohorts.
$\mathrm{Ma}$ et al. reported that $\geq 1 \%$ PD-L1 expression was present in $40 \%$ TC and in $22.2 \%$ TILs, while no effect on OS or DFS was shown (23). Such a low expression rate of PD-L1 has also been observed in some retrospective studies, with levels of 20$40 \%(18,24-26)$. On the other hand, several retrospective studies have reported $\mathrm{PD}-\mathrm{L} 1$ positivity and high $\mathrm{PD}-\mathrm{L} 1$ expression rates in up to $70-100$ and $34-46 \%$ of NPC tumor samples, respectively $(14,16,17,27,28)$. In our analysis, the PD-L1 positivity rate was $83 \%$, which is in line with existing literature and similar to that in an Asian population.

There could be multiple additional explanations for the discrepancies regarding $\mathrm{PD}-\mathrm{L} 1$ expression reported in the literature. Notably, no uniform antibody was used for IHC staining for PD-L1 and staining protocols differed markedly in the above-mentioned studies. In fact, a recent work highlighted that PD-L1 IHC staining results differ strongly depending on the antibody utilized (29). Apart from that, no validated PDL1 scoring algorithm for NPC has so far been developed and there is no widely accepted consensus regarding the cut off values for PD-L1 positivity. Of note, we used the PD-L1 (clone 22C3) mouse monoclonal antibody from Dako, which is included in the FDA approved diagnostic tool for PD-L1 staining in various solid malignancies.

The expression of PD-L1 in NPC is upregulated by EBVinduced latent membrane protein 1 (LMP1): In an in vitro study, EBV-negative cell lines showed lower PD-L1 expression than EBV-positive cell lines (14). According to literature, 90\% of endemic NPCs in southeast Asia are EBV-associated (30). In our Caucasian cohort, only $63 \%$ of the patients showed EBV 
TABLE 4 | Studies investigating the association of outcome parameters and PD-L1 expression in NPC.

\begin{tabular}{|c|c|c|c|c|c|c|}
\hline References & $n$ & PD-L1 antibody & Cells & Cut-off values & $n$ per group & Outcome \\
\hline Zhou et al. (16) & 115 & E1L3N, cell signaling & Tumor & H-score $<115, \geq 115$ & 38,61 & Worse OS \\
\hline \multirow[t]{2}{*}{ Zhang et al. (17) } & 160 & E1L3N, cell signaling & Tumor & H-score $\leq 35,>35$ & 69,58 & Worse DFS \\
\hline & & & TILs & - & - & - \\
\hline Lee et al. (18) & 160 & E1L3N, cell signaling & Tumor & $<5,5-24, \geq 25 \%$ & $78,4,22$ & Better LRFS, PFS \\
\hline \multirow[t]{2}{*}{ Qu et al. (24) } & 96 & ab205921, abcam & Tumor & $\leq 10,>10 \%$ & 68,28 & Worse DMFS \\
\hline & & & TILs & - & - & - \\
\hline \multirow[t]{2}{*}{ Chan et al. (26) } & 161 & SP142, Ventana & Tumor & $<1,1-4, \geq 5 \%$ & $122,39,24$ & No correlation with PFS, OS \\
\hline & & & TILs & $<1,1-4, \geq 5 \%$ & $40,121,38$ & No correlation with PFS, OS \\
\hline Zheng et al. (28) & 116 & E1L3N, cell signaling & Tumor & $0-50,>50 \%$ & 87,29 & Worse OS \\
\hline \multirow[t]{2}{*}{ Ono et al. (3) } & 66 & D3, cell signaling & Tumor & $0-4, \geq 5 \%$ & 13,53 & No correlation with PFS, OS \\
\hline & & & TILs & $0-4, \geq 5 \%$ & 16,50 & No correlation with PFS, OS \\
\hline
\end{tabular}

n, Total number of patients; LRFS, local recurrent free survival; PFS, progression free survival; OS, overall survival; DMFS, distant metastases free survival.

association, and in line with this observation, only a minority of the tumors (20\%) showed high PD-L1 expression on tumor cells. This probably reflects the high proportion of EBV-negative patients, who harbor a markedly different immunological tumor microenvironment from EBV-positive patients (31).

Multiple conflicting reports regarding the prognostic role of PD-L1 expression on NPC TC have been published for Asian patients, although the interpretation of these results has to be performed with caution, since different antibodies, staining techniques, and inclusion criteria were utilized. Zheng et al. proposed that a higher PD-L1 expression on TC translates into worse OS (28). In contrast, two other studies did not report a correlation of survival with PD-L1 expression on TC of NPC patients $(3,26)$ and one study even proposed an improvement of OS with higher PD-L1 expression (18). Additionally, in a cohort of 132 Asian NPC patients, higher PD-L1 expression translated into a worse prognosis in terms of DFS. Furthermore, the distant metastasis free survival was poorer in patients with high PD-L1 expression, as shown in a study of 99 NPC patients (24) (overview summarized in Table 4). Very recently, a meta-analysis on the prognostic significance of PD-L1/PD-1 in NPC patients has been published (32). This study shows that the PD-L1/PD-1 detection methods and cut-off values differed markedly between the analyzed reports and no correlation of PD-L1/PD-1 expression with survival in Asian NPC patients was demonstrated (32).

However, another recent study from the Netherlands, which presumably included a high proportion of Caucasian patients in addition to Asian patients, was not included in the aforementioned meta-analyses, but probably better reflects our patient cohort. This study showed a strong association between a better DFS with a higher rate of CD8 infiltration of TILs and
PD-L1-positive TILs, which confirms our findings (31). Other potential immune biomarkers such as PD-1 expression on TILs did not have a significant impact on the survival parameters, which is in accordance with existing literature (16). The role of LAG3 expression on TC in NPC remains elusive since we are not aware of any reports evaluating the role of LAG3 in Asian patients and did not find its association with OS and DFS in our Caucasian cohort study.

In conclusion, NPC is an orphan disease in the western world and the vast majority of molecular insights and clinical data on this disease originate from the endemic regions in southeast Asia. Given the limitations of small sample size and the retrospective nature of our analysis, we found that PD-L1 expression on TC and TILs in a Caucasian population is associated with OS and DFS, respectively. A prospective biomarker analysis of a predefined Caucasian NPC subpopulation would be desirable in future trials.

\section{DATA AVAILABILITY STATEMENT}

All datasets generated for this study are included in the article/supplementary material.

\section{ETHICS STATEMENT}

The studies involving human participants were reviewed and approved by Ethics committee of the Medical University of Vienna (EK 1416/2017). Written informed consent for participation was not required for this study in accordance with the national legislation and the institutional requirements. 


\section{AUTHOR CONTRIBUTIONS}

Study design was planned and preparation and editing of the manuscript was done by CM, TF, and FO. Data acquisition was

\section{REFERENCES}

1. Chang ET, Adami HO. The enigmatic epidemiology of nasopharyngeal carcinoma. Cancer Epidemiol Biomarkers Prev. (2006) 15:1765-77. doi: 10.1158/1055-9965.EPI-06-0353

2. Lee AW, Foo W, Mang O, Sze WM, Chappell R, Lau WH, et al. Changing epidemiology of nasopharyngeal carcinoma in Hong Kong over a 20-year period (1980-99): an encouraging reduction in both incidence and mortality. Int J Cancer. (2003) 103:680-5. doi: 10.1002/ijc.10894

3. Ono T, Azuma K, Kawahara A, Sasada T, Matsuo N, Kakuma T, et al. Prognostic stratification of patients with nasopharyngeal carcinoma based on tumor immune microenvironment. Head Neck. (2018) 40:2007-19. doi: $10.1002 /$ hed.25189

4. Chua MLK, Wee JTS, Hui EP, Chan ATC. Nasopharyngeal carcinoma. Lancet. (2016) 387:1012-24. doi: 10.1016/S0140-6736(15)0 0055-0

5. Vaughan TL, Shapiro JA, Burt RD, Swanson GM, Berwick M, Lynch CF, et al. Nasopharyngeal cancer in a low-risk population: defining risk factors by histological type. Cancer Epidemiol Biomarkers Prev. (1996) 5: 587-93.

6. Müller E, Beleites E. The basaloid squamous cell carcinoma of the nasopharynx. Rhinology. (2000) 38:208-11. doi: 10.1002/10970142(19951115)76:10<1689::aid-cncr2820761003>3.0.co;2-9

7. Roy C, Choudhury KB, Basu S. Basaloid squamous cell carcinoma of nasopharynx: an extremely rare variety of tumour of nasopharynx. J Indian Med Assoc. (2011) 109:343-4.

8. Wei WI, Sham JS. Nasopharyngeal carcinoma. Lancet. (2005) 365:2041-54. doi: 10.1016/S0140-6736(05)66698-6

9. NCCN Clinical Practice Guidelines in Oncology: Head and Neck Cancers. (2019). Available online at: https://www.nccn.org/professionals/ physician_gls/pdf/head-and-neck.pdf

10. Zhang Y, Chen L, Hu GQ, Zhang N, Zhu XD, Yang KY, et al. Gemcitabine and cisplatin induction chemotherapy in nasopharyngeal carcinoma. $N$ Engl $J$ Med. (2019) 381:1124-35. doi: 10.1056/NEJMoa1905287

11. Hamilton SN, Ho C, Laskin J, Zhai Y, Mak P, Wu J. Asian versus non-Asian outcomes in nasopharyngeal carcinoma: a North American population-based analysis. Am J Clin Oncol. (2016) 39:575-80. doi: 10.1097/COC.0000000000000091

12. Khanna R, Busson P, Burrows SR, Raffoux C, Moss DJ, Nicholls $\mathrm{JM}$, et al. Molecular characterization of antigen-processing function in nasopharyngeal carcinoma (NPC): evidence for efficient presentation of Epstein-Barr virus cytotoxic T-cell epitopes by NPC cells. Cancer Res. (1998) 58:310-4.

13. Pai S, O'Sullivan B, Abdul-Jabbar I, Peng J, Connoly G, Khanna R, et al. Nasopharyngeal carcinoma-associated Epstein-Barr virus-encoded oncogene latent membrane protein 1 potentiates regulatory T-cell function. Immunol Cell Biol. (2007) 85:370-7. doi: 10.1038/sj.icb. 7100046

14. Fang W, Zhang J, Hong S, Zhan J, Chen N, Qin T, et al. EBV-driven LMP1 and IFN-gamma up-regulate PD-L1 in nasopharyngeal carcinoma: implications for oncotargeted therapy. Oncotarget. (2014) 5:12189-202. doi: $10.18632 /$ oncotarget.2608

15. Zhou Y, Miao J, Wu H, Tang H, Kuang J, Zhou X, et al. PD-1 and PD-L1 expression in 132 recurrent nasopharyngeal carcinoma: the correlation with anemia and outcomes. Oncotarget. (2017) 8:51210-23. doi: 10.18632/oncotarget.17214

16. Zhou Y, Shi D, Miao J, Wu H, Chen J, Zhou X, et al. PD-L1 predicts poor prognosis for nasopharyngeal carcinoma irrespective of PD-1 and EBV-DNA load. Sci Rep. (2017) 7:43627. doi: 10.1038/sr ep43627 performed by TF, CM, FO, CW, and GK. Quality control of data algorithms and data analysis was performed by TF, CM, FO, LM, and CK. The statistical analysis was done by CK. Review of the manuscript was by TF, CM, FO, LM, CW, GK, and CK.

17. Zhang J, Fang W, Qin T, Yang Y, Hong S, Liang W, et al. Coexpression of PD-1 and PD-L1 predicts poor outcome in nasopharyngeal carcinoma. Med Oncol. (2015) 32:86. doi: 10.1007/s12032-0150501-6

18. Lee VH, Lo AW, Leung CY, Shek WH, Kwong DL, Lam KO, et al. Correlation of PD-L1 expression of tumor cells with survival outcomes after radical intensity-modulated radiation therapy for nonmetastatic nasopharyngeal carcinoma. PLoS ONE. (2016) 11:e0157969. doi: 10.1371/journal.pone.0157969

19. Cohen EEW, Soulières D, Le Tourneau C, Dinis J, Licitra L, Ahn MJ, et al. Pembrolizumab versus methotrexate, docetaxel, or cetuximab for recurrent or metastatic head-and-neck squamous cell carcinoma (KEYNOTE-040): a randomised, open-label, phase 3 study. Lancet. (2019) 393:156-67. doi: 10.1016/S0140-6736(18) 31999-8

20. Fehrenbacher L, Spira A, Ballinger M, Kowanetz M, Vansteenkiste J, Mazieres J, et al. Atezolizumab versus docetaxel for patients with previously treated non-small-cell lung cancer (POPLAR): a multicentre, open-label, phase 2 randomised controlled trial. Lancet. (2016) 387:1837-46. doi: 10.1016/S0140-6736(16) 00587-0

21. Karpathiou G, Casteillo F, Giroult JB, Forest F, Fournel P, Monaya A, et al. Prognostic impact of immune microenvironment in laryngeal and pharyngeal squamous cell carcinoma: Immune cell subtypes, immuno-suppressive pathways and clinicopathologic characteristics. Oncotarget. (2017) 8:19310-22. doi: 10.18632/oncotarget. 14242

22. Zhang Y, Liu YD, Luo YL, Liu BL, Huang QT, Wang F, et al. Prognostic value of lymphocyte activation gene-3 (LAG-3) expression in esophageal squamous cell carcinoma. J Cancer. (2018) 9:4287-93. doi: 10.7150/jca. 26949

23. Ma BBY, Lim WT, Goh BC, Hui EP, Lo KW, Pettinger A, et al. Antitumor activity of nivolumab in recurrent and metastatic nasopharyngeal carcinoma: an international, multicenter study of the mayo clinic phase 2 consortium (NCI-9742). J Clin Oncol. (2018) 36:1412-8. doi: 10.1200/JCO.2017. 77.0388

24. Qu Y, Wang D, Yang L, Liu HY, Cui W, Che YQ. Expression and clinical significance of programmed death ligand 1 in nasopharyngeal carcinoma. Mol Clin Oncol. (2018) 9:75-81. doi: 10.3892/mco.20 18.1633

25. Zhu Q, Cai MY, Chen CL, Hu H, Lin HX, Li M, et al. Tumor cells PD-L1 expression as a favorable prognosis factor in nasopharyngeal carcinoma patients with pre-existing intratumor-infiltrating lymphocytes. Oncoimmunology. (2017) 6:e1312240. doi: 10.1080/2162402X.2017. 1312240

26. Chan OS, Kowanetz M, Ng WT, Koeppen H, Chan LK, Yeung RM, et al. Characterization of PD-L1 expression and immune cell infiltration in nasopharyngeal cancer. Oral Oncol. (2017) 67:52-60. doi: 10.1016/j.oraloncology.2017. 02.002

27. Hsu MC, Hsiao JR, Chang KC, Wu YH, Su IJ, Jin YT, et al. Increase of programmed death-1-expressing intratumoral CD8 T cells predicts a poor prognosis for nasopharyngeal carcinoma. Mod Pathol. (2010) 23:1393-403. doi: 10.1038/modpathol.2010.130

28. Zheng LF, Cao CN, Cheng GP, Hu QY, Chen XZ. Cytomembranic PD-LI expression in locoregionally advanced nasopharyngeal carcinoma. Oncotargets Ther. (2017) 10:5483-7. doi: 10.2147/OTT.S 152007

29. Büttner R, Gosney JR, Skov BG, Adam J, Motoi N, Bloom KJ, et al. Programmed death-ligand 1 immunohistochemistry testing: a review 
of analytical assays and clinical implementation in non-small-cell lung cancer. J Clin Oncol. (2017) 35:3867-76. doi: 10.1200/JCO.2017. 74.7642

30. Lo YM, Leung SF, Chan LY, Chan AT, Lo KW, Johnson PJ, et al. Kinetics of plasma Epstein-Barr virus DNA during radiation therapy for nasopharyngeal carcinoma. Cancer Res. (2000) 60:2351-5.

31. Ooft ML, van Ipenburg JA, Braunius WW, Zuur CI, Koljenović S, Willems SM. Prognostic role of tumor infiltrating lymphocytes in EBV positive and EBV negative nasopharyngeal carcinoma. Oral Oncol. (2017) 71:16-25. doi: 10.1016/j.oraloncology.2017. 05.015

32. Huang ZL, Liu S, Wang GN, Zheng SH, Ding SR, Tao YL, et al. The prognostic significance of PD-L1 and PD-1 expression in patients with nasopharyngeal carcinoma: a systematic review and metaanalysis. Cancer Cell Int. (2019) 19:141. doi: 10.1186/s12935-019-0 863-5
Conflict of Interest: TF received travel grants from: MSD, BMS, Merck, and Roche. TF is in advisory boards for: MSD, Merck, Astra Zeneca, Roche, and BI; and received honoraria from: MSD, BMS, Roche, Accord, Sanofi, BI, Novartis, and Astra Zeneca. CM received travel grants from: Roche, BMS, MSD, and Merck; and honoraria from BI and Merck.

The remaining authors declare that the research was conducted in the absence of any commercial or financial relationships that could be construed as a potential conflict of interest.

Copyright $(02019$ Minichsdorfer, Oberndorfer, Krall, Kornek, Müllauer, Wagner and Fuereder. This is an open-access article distributed under the terms of the Creative Commons Attribution License (CC BY). The use, distribution or reproduction in other forums is permitted, provided the original author(s) and the copyright owner(s) are credited and that the original publication in this journal is cited, in accordance with accepted academic practice. No use, distribution or reproduction is permitted which does not comply with these terms. 